J. Dairy Sci. 94:6288-6289

(C) American Dairy Science Association, 2011

\title{
2011 ADSA Undergraduate Scholarship Recognition Awards
}

\author{
Sophomores \\ (Bronze certificate) \\ Taylor Pires \\ Erin DeSnayer \\ Eric Diehl \\ Elizabeth Fullerton \\ Sara King \\ Corey Reed \\ Matthew Sharpe \\ Adam Shuknecht
}

Matthew Scott

Katelyn Horning

Heidi Moff

\author{
Juniors \\ (Silver certificate)
}

\author{
Seniors \\ (Gold certificate)
}

California Polytechnic State University; San Luis Obispo

David Vander Schaaf

W. David Jones

Cornell University

Larry Bardroff

Carolyn Braun

Katie Donnan

Daniel Durfee

Jordan Fisher

Ariel Garland

James Hunt

Jesse Johnson

Julia Knight

Bethany Liebig

Daniel Lopez

Zachary Mason

Kendra Murray

Brianna Pomeroy

Iowa State University

Brianna Adamic

Emily Benedict

Elizabeth Coughlin

Jessica Engelking

Erich Hodges

Louisiana State University

Stephanie Thibeau

Michigan State University

Kelsey DeLand

Caroline Szybisty

\section{North Carolina State University}

Kristen Glosson

Laura Such

The Ohio State University

Laura Gordon

Melinda Miller
Christina Puetz

Mark Fagundes

Sarah Barber

Carlie Brown

Samuel Fessenden

Lucas Fuess

Shelley Honda

Scott Laca

Jason McNamara

Ashley Sears

Ashley Severy

Amanda Smith

Thomas Smith

Amanda Stang

Eve Waters

Emily Ullerich

Brittany Charpentier

Hayley Hollier

Emily Harris

Jesica Leonard

Jason Hartschuh

Alissa Hunter

Andrew Lefeld

Jason Miley 
Nathan Ulmer

Blaine Melody

Jacob Achen

Isaac Salfer

Kelsey Smith Brittany Trexler

Sarah Williams
The Pennsylvania State University

Philip Bachman

Joshua Ebert

Laraya Foresman

Kolby McIntyre

\section{University of Illinois}

$\begin{array}{cc} & \text { Emily Lyons } \\ \text { Cassandra Folkerts } & \text { Loren Heusel } \\ \text { University of Minnesota } & \\ \text { Jason Kaare } & \text { Laura Weisz } \\ & \text { Michael Donnelly }\end{array}$

Virginia Polytechnic Institute and State University

Sarah Burczynski

Erin Henley

Lindsay Hetrick

Lauryn Singer
Dana Gochenour

Brittany Thompson

Holley Weeks 